Planetary Systems in the Universe - Observation, Formation and Evolution

Proceedings IAU Symposium No. 202, (c)2004 IAU

Alan Penny, Pawel Artymowicz, Anne-Marie Lagrange, 8 Sara Russell, eds.

\title{
Planetary Messages in the Doppler Residuals
}

\author{
Geoffrey W. Marcy \\ Astronomy Dept., University of California, Berkeley, CA 94720 USA
}

Debra A. Fischer

Astronomy Dept., University of California, Berkeley, CA 94720 USA

R. Paul Butler

Dept. of Terrestrial Magnetism, Carnegie Inst. of Washington, 5243 Broad Branch Rd, NW, Washington DC, 20015-1305 USA

\author{
Steven S. Vogt \\ UCO/Lick Observatories, University of California, Santa Cruz, CA \\ $95064 U S A$
}

\begin{abstract}
The Doppler residuals to the Keplerian fits for extrasolar planets reveal important properties of the planets and host stars. Stellar magnetic fields modify the photospheric velocity fields, causing Doppler fluctuations with unknown time scales. This Doppler "jitter", seen prominently in the magnetic stars Epsilon Eridani and $\xi$ Boo A, compromises the detectability of planets. The Doppler residuals during the transit of HD209458 reveal that the planet orbits in the same direction as the star spins. Moreover, the transit path across the star is nearly parallel to the stellar equator. Most interestingly, the Doppler residuals of known planets often reveal additional coherent variations, probably caused by additional companions. Both 55 Cancri and HD168443 reveal such coherent Doppler residuals. Another five planet-bearing stars observed at Lick show trends in the Doppler residuals indicating the presence of additional companions. Remarkably, about half of the known extrasolar planets reveal such coherent variations. This suggests that stars with planets have a high occurrence rate of harboring more distant companions, planetary or otherwise.
\end{abstract}

\section{Introduction}

Currently, 50 extrasolar planet candidates have been identified, all having masses probably under $13 \mathrm{M}_{\mathrm{JUP}}$ (see Butler et al. and Udry et al., this volume). To avoid false detections, the Doppler amplitudes are typically 5-10× larger than the Doppler errors (Marcy, Cochran, Mayor 2000). The historical record shows that false claims often result from wobble amplitudes that are comparable to the scatter in the Keplerian fit (Doppler or astrometric).

As a bonus, the high signal-to-noise Keplerian fits imbue the Doppler residuals themselves with useful information about additional companions and stellar effects. We study the Doppler residuals in the 30 planet candidates found to 

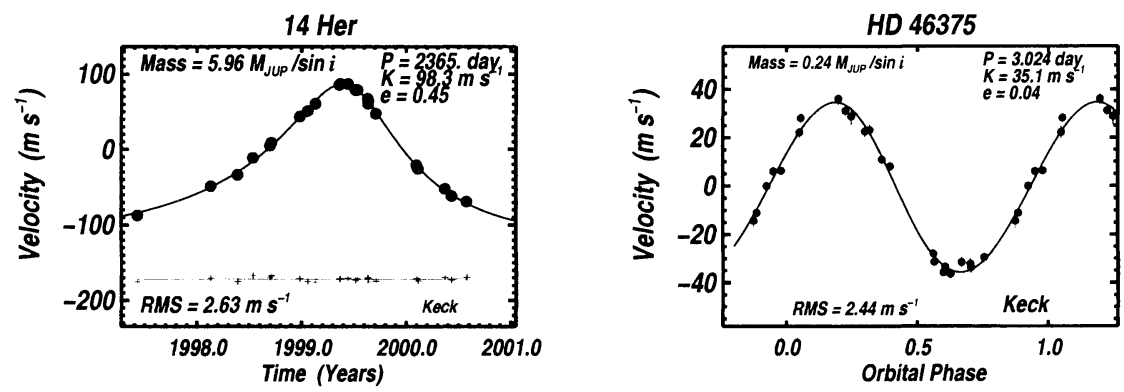

Figure 1. Integrity of Doppler residuals in detecting additional planets. Left: Doppler measurements for $14 \mathrm{Her}$ (K0V) showing the Keplerian curve (solid line) due to a planet that has $P=6.5 \mathrm{yr}$ and semimajor axis, $a=3.5$ AU. The residuals (crosses) scatter with $\mathrm{RMS}=2.6 \mathrm{~m} \mathrm{~s}^{-1}$, implying that no additional companions of Jupiter-mass $(M \sin i)$ exist within $5 \mathrm{AU}$, nor of saturn-mass within 0.5 AU. Right: Doppler measurements for HD46375 (K1IV) versus orbital phase. The planet has $M \sin i=0.8 \mathrm{M}_{\mathrm{SAT}}$, in an orbit having $P=3.024 \mathrm{~d}$ and semimajor axis, $a=0.041 \mathrm{AU}$. The residuals scatter with $\mathrm{RMS}=2.44 \mathrm{~m} \mathrm{~s}^{-1}$, consistent with errors, and rule out additional companions of Jupiter-mass $(M \sin i)$ within $2 \mathrm{AU}$ and saturn-mass within 0.5 AU.

date from our survey of 1,100 FGKM main sequence stars observed at the Lick, Keck, and AAT telescopes (Butler et al. 1999). Our typical Doppler precision of $3 \mathrm{~m} \mathrm{~s}^{-1}$ permits a search for subtle effects in the residuals. Here we discuss three interesting sources of these Doppler residuals: photospheric activity, transits, and additional companions.

\section{Stars Showing Pure Keplerian Behavior}

One may assess true Doppler errors empirically, by the constancy of the velocities of the chromosphericaly quiet stars. Approximately half of our 1,100 FGKM target stars exhibit a Doppler RMS of less than $5 \mathrm{~m} \mathrm{~s}^{-1}$, indicative of our errors and and stellar "noise". However, the remaining $50 \%$ of our stars show Doppler variations above $5 \mathrm{~m} \mathrm{~s}^{-1}$ rendering these variations intrinsic to the star and/or companions, rather than errors.

Representative standard stars (with no companions) are shown for $\sim 30$ FGKM stars in Vogt et al. (2000) and Fischer et al. (2000). These show the typical Doppler scatter of $5 \mathrm{~m} \mathrm{~s}^{-1}$ consistent with errors. Similarly, stars harboring one dominant planet exhibit pure Keplerian variations, with residuals that scatter by $\sim 5 \mathrm{~m} \mathrm{~s}^{-1}$, as expected from errors. Additional planets are revealed by residuals that exceed errors.

Two representative cases of stars that have only a single dominant planet are shown in Figure 1. Our Doppler measurements from Keck for $14 \mathrm{Her}$ (K0V) clearly show the planet with minimum mass of $5.9 \mathrm{M}_{\mathrm{JUP}}$ and orbital period, $P=6.5 \mathrm{yr}$, giving a semimajor axis, $a=3.5 \mathrm{AU}$. The velocity residuals exhibit an RMS of $2.6 \mathrm{~m} \mathrm{~s}^{-1}$ during the 4 years, with no apparent systematic departures. Thus, one rules out companions having $M \sin i>1 \mathrm{M}_{\mathrm{JUP}}$ within $5 \mathrm{AU}$, as such 


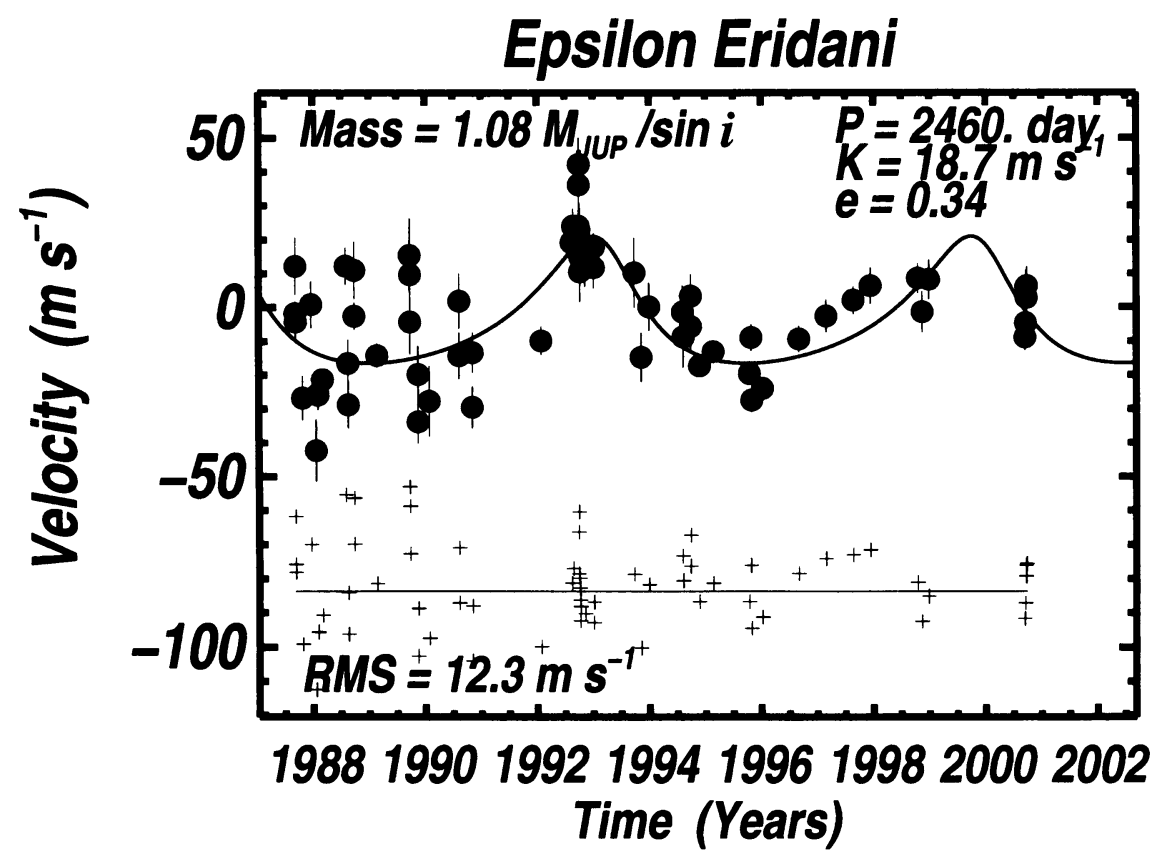

Figure 2. Doppler jitter of $15-20 \mathrm{~m} \mathrm{~s}^{-1}$, caused by magnetic activity in FGK stars younger than 1 Gyr. The 1 Gyr-old star, $\epsilon$ Eri, exhibits jitter of $15 \mathrm{~m} \mathrm{~s}^{-1}$. Subtraction of a possible orbit (solid line) leaves residuals (crosses) that have RMS of $12.3 \mathrm{~m} \mathrm{~s}^{-1}$, far greater than errors (see error bars). The credibility of the Keplerian variation is weakened by Doppler jitter, as is the detectability of additional planets.

companions would produce residuals of $\sim 10 \mathrm{~m} \mathrm{~s}^{-1}$. Note that a companion with mass $1 \mathrm{M}_{\mathrm{JUP}}$ or less that orbits within $1 \mathrm{AU}$ would probably be dynamically stable (Rivera \& Lissauer 2000, Laughlin and Adams 1999), and thus can't be ruled out. Nonetheless, 14 Her harbors a companion having a mass of at least $6 \mathrm{M}_{\mathrm{JUP}}$ at 3.5 $\mathrm{AU}$, but has no other Jupiter-mass companions within $5 \mathrm{AU}$, based on the flat residuals. Moreover, the lack of a Doppler trend rules out a brown dwarf companion (10-80 MJUP) within $\sim 25 \mathrm{AU}$.

A similar lack of additional planetary companions is revealed for HD46375 (Figure 1) for which the Doppler residuals of $2.4 \mathrm{~m} \mathrm{~s}^{-1}$ argue against the presence of Jupiter-mass companions within several AU. This case is intriguing, as the planet has $M \sin i$ less than the mass of Saturn. Yet, there is no apparent additional companion to perform the gravitational scattering of that saturn inward to its current location (eg., Levison et al. 1998, Weidenschilling and Marzari 1996). If such a scattering culprit exists, it either orbits farther than 3 $\mathrm{AU}$ or it has mass under $\sim 1 \mathrm{M}_{\mathrm{SAT}}$, both possibilities rendering it undetectable with present data. 


\section{Velocity Jitter in Young Stars}

Magnetic fields on the surfaces of stars cause fluctuations in photospheric hydrodynamics, producing variable Doppler shifts. Several studies have demonstrated an increase in Doppler variations ("jitter") with increasing magnetic fields (e.g., Saar et al. 1998, Saar \& Fischer 2000, Santos this volume), using the chromospheric emission from $\mathrm{Ca}^{+}$at $3933 \AA$ as the standard magnetic proxy. Main sequence stars of spectral type $\mathrm{F}, \mathrm{G}$ and $\mathrm{K}$, that are younger than 1 Gyr show magnetic-induced Doppler variations of $10-20 \mathrm{~m} \mathrm{~s}^{-1}$ (Cumming et al. 1999, Saar et al. 1998). However, from the late K and M-type main sequence stars, the Doppler jitter diminishes from $10 \mathrm{~m} \mathrm{~s}^{-1}$ to under $5 \mathrm{~m} \mathrm{~s}^{-1}$ for magnetic stars.

Doppler measurements from a typical young K dwarf, $\epsilon$ Eri (age = $1 \mathrm{Gyr}$ ), are shown in Figure 2. Its velocity variations of $20 \mathrm{~m} \mathrm{~s}^{-1}$ during 12 years greatly exceed the errors of $3 \mathrm{~m} \mathrm{~s}^{-1}$. Even after removal of a possible Keplerian curve (Cumming et al. 1999; Hatzes et al. 2000), the residuals have RMS $=12 \mathrm{~m} \mathrm{~s}^{-1}$ (Figure 2), caused by changing photospheric velocity fields. Similarly, the young magnetic star, $\xi$ Boo A (G8V) shows velocity jitter of $31 \mathrm{~m} \mathrm{~s}^{-1}$ (RMS) (Saar and Fischer 2000). The time scales of the velocity jitter for young $\mathrm{G}$ and $\mathrm{K}$ dwarfs remain unknown, and periods of years are plausibly similar to known magnetic time scales (dynamo, spot cycles). Therefore, Doppler amplitudes under 50 $\mathrm{m} \mathrm{s}^{-1}$ could be caused by photospheric variations or by planets. Thus, the interpretation of periodicities in the velocities of young $\mathrm{G}$ and $\mathrm{K}$ stars such as $\epsilon$ Eri remains ambiguous.

The error budget should include photospheric Doppler jitter alongside photonlimited measurement errors, which themselves are enhanced due to rotational Doppler broadening of spectral lines in young stars. Thus FGK stars that are younger than 1 Gyr exhibit Doppler variations of $\sim 15-20 \mathrm{~m} \mathrm{~s}^{-1}$. Such jitter hinders the detection of planets and imposes a challenge to planet detection in young clusters such as the Pleiades and Hyades (see Cochran et al. this volume).

\section{Planet Transits: Doppler effects}

During the planet transit of the star HD209458 (Henry et al. 2000, Charbonneau et al. 2000), the measured Doppler shift varies due to the planet occulting the approaching and receding portions of the stellar hemisphere (Queloz et al. 2000 ). The equatorial rotation velocity of $\sim 4 \mathrm{~km} \mathrm{~s}^{-1}$ coupled with the occulted stellar area of $1.6 \%$, causes the $40 \mathrm{~m} \mathrm{~s}^{-1}$ deviation when the planet is at the stellar limb. We have measured this effect using Keck HIRES spectra, with a precision of $\pm 3 \mathrm{~m} \mathrm{~s}^{-1}$ as shown in Figure 3. Just after first contact of the transit, the spectrum is redshifted which shows that the occulted limb was approaching. Hence the star is spinning in the same direction as the planet orbits around the star, in agreement with Queloz et al. (2000). Interestingly, the velocity crosses zero just prior to the midpoint of the transit as seen photometrically. This shows that the transit path of the planet is bisected by the spin axis of the star, implying that the planet's transit path is nearly parallel 


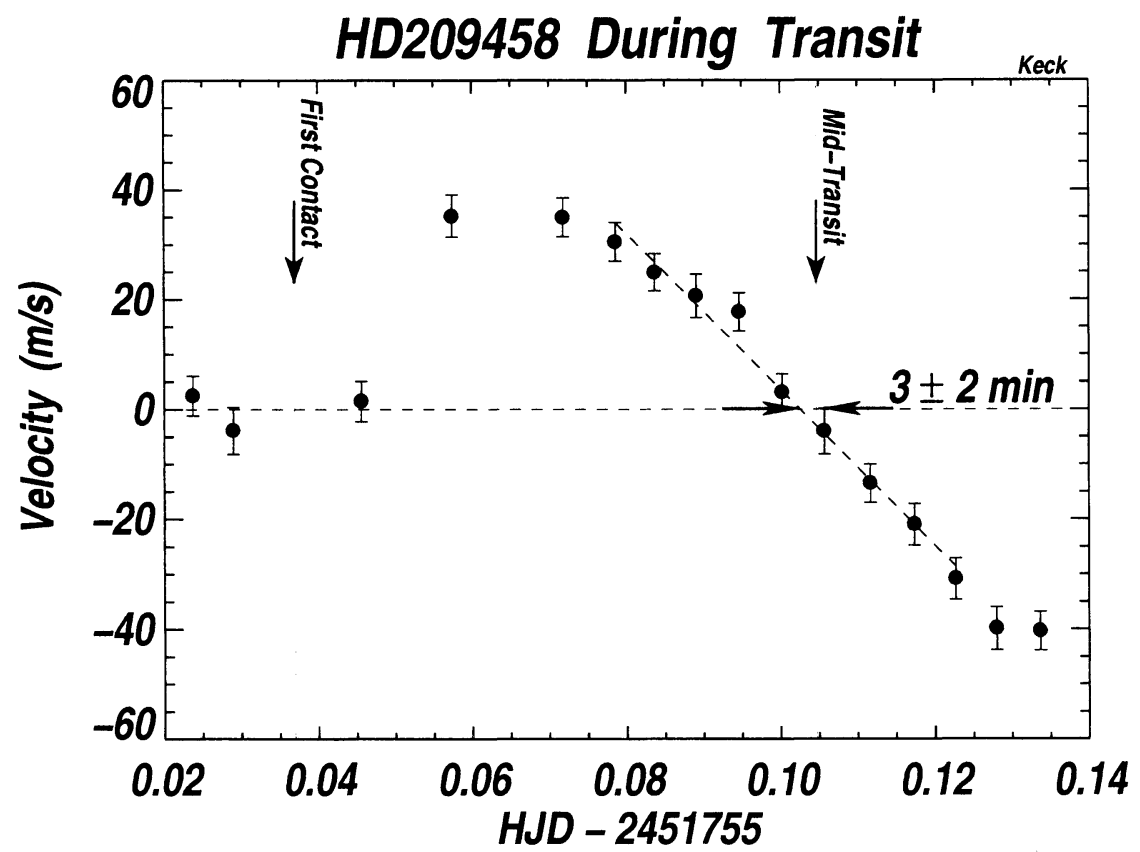

Figure 3. Velocities during the planet transit of HD209458 (G0V). The rise and fall are caused by the planet occulting the approaching and receding surface of the rotating star. The net redshift during the first half of the transit shows that the planet orbits in the same direction as the star spins. Moreover, the velocities cross zero just before the midpoint of the photometric transit, implying that the path of the planet is nearly parallel to the stellar equator. The slight asymmetry in the velocity amplitudes, $+35 \mathrm{~m} \mathrm{~s}^{-1}$ and $-40 \mathrm{~m} \mathrm{~s}^{-1}$, similarly implies a path that is nearly, but not exactly, parallel to the equator, in agreement with Queloz et al (2000).

with stellar equator. This conclusion is further supported by the symmetry of the positive and negative excursions of the velocities during transit.

We further searched for spectral features, absorption or emission, caused by starlight passing through the planet's atmosphere during the transit (Bundy and Marcy 2000). No such features were found across the optical spectrum at detectable levels of a few percent. Further work on the Doppler variations and spectral features during transit will be carried out by Dave Charbonneau, Bob Noyes, and Tim Brown, using these Keck spectra.

\section{Coherent Doppler Residuals: Additional Orbiting Companions}

We have carried out a study of the planet-bearing stars discovered at Lick Observatory (Fischer et al. 2000). Nearly $50 \%$ of them reveal Doppler residuals to the Keplerian fit that vary coherently with time, indicating the presence of additional companions. 


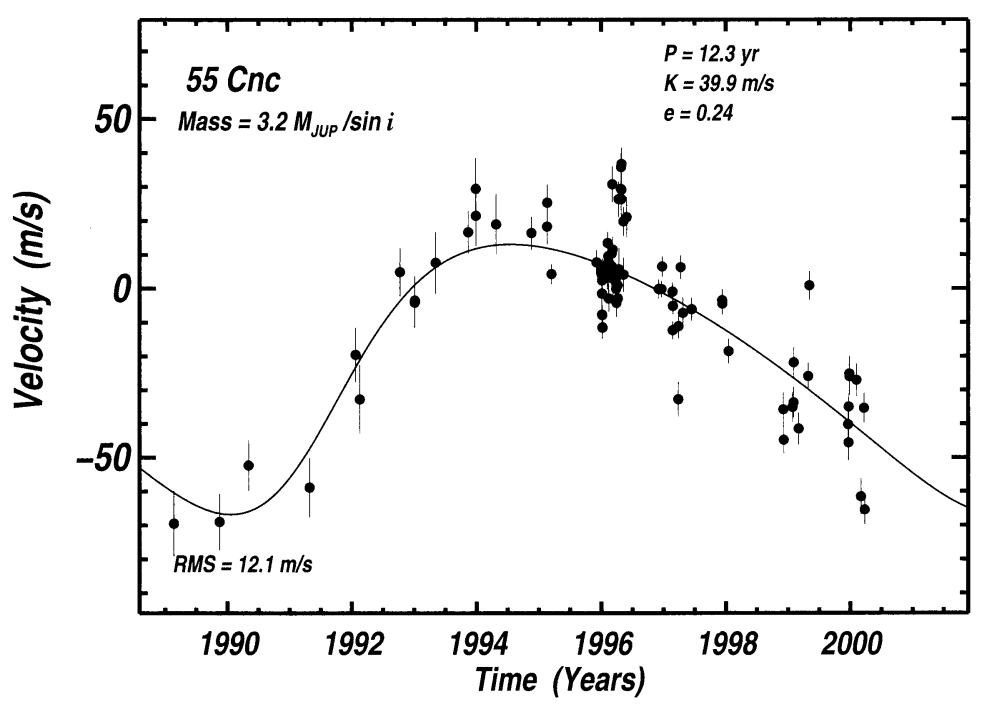

Figure 4. Residual velocities for 55 Cancri (G8V), obtained by subtracting the known Keplerian orbit of the inner planet $(\mathrm{P}=14.6 \mathrm{~d})$ from the measured velocities. The residual velocities are consistent with a Keplerian orbit representing a second planet having a mass of $3.2 \mathrm{M}_{\mathrm{JUP}} / \sin i$ and a period of, $\mathrm{P}=12.3 \mathrm{yr}$, implying a semimajor axis of $5.1 \mathrm{AU}$. However, this outer companion requires another year of velocity measurements to be sure that the velocities complete a full orbit.

Four stars from Lick were recently revealed to have residual velocity variations consistent with the presence of additional distant companions: HD217107, 55 Cancri, $\tau$ Boo, and HD 38529 (Fischer et al. 2000). For $\tau$ Boo, an M2V stellar companion resides 2.8 arcsec (42 AU) away and may be partly responsible for the Doppler residuals (see Lloyd et al. this volume). Upsilon Andromedae had previously revealed coherent residuals and is now known to have 3 planets (Butler et al. 1999). GJ 876 exhibits Doppler residuals that are only quasicoherent, leaving us puzzled about their origin (Fischer et al. 2000). Surface magnetic activity remains a viable explanation.

\subsection{Cancri}

The star, 55 Cnc, has a known inner planet with orbital period of $14.65 \mathrm{~d}$ (Butler et al. 1996), and the trend in the residuals was displayed by Marcy and Butler (1998). New velocities reveal residuals shown in Figure 4, which are consistent with a second planetary-mass companion having $M \sin i=3.2 \mathrm{M}_{\mathrm{JUP}} / \sin i$, an orbital period of $12.3 \mathrm{yr}$, and a semimajor axis, $a=5.1 \mathrm{AU}$.

This outer companion, if real, is the first extrasolar planet that resides at the same distance as Jupiter in the Solar System. If so, its orbit is apparently eccentric.

The eccentric orbit for the prospective component "c" orbiting 55 Cancri provides the first empirical indication that some planets at 5 AU reside in 


\section{HD168443}

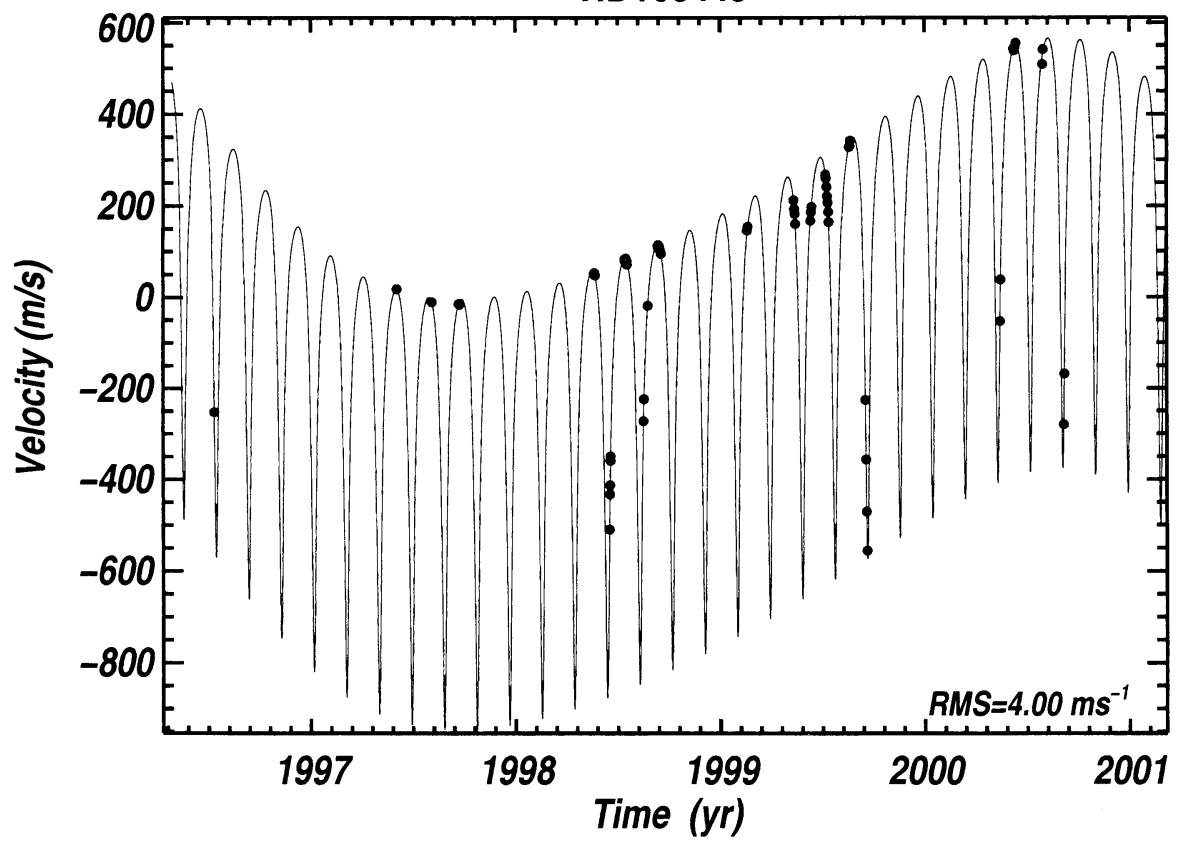

Figure 5. Velocities for HD168443 (G5IV), fit well with a Keplerian model containing two companions (solid line). The two companions have $M \sin i$ $=6.6 \mathrm{M}_{\mathrm{JUP}}$ and $16 \mathrm{M}_{\mathrm{JUP}}$, at semimajor axes of 0.30 and $2.7 \mathrm{AU}$, respectively. The outer companion appears to be a "brown dwarf" in a system with another companion of perhaps less mass. The dynamical stability of the two companions within $3 \mathrm{AU}$ suggests that their masses are less than $\sim 60 \mathrm{M}_{\text {JUP }}$ (Laughlin, personal comm., 2000).

eccentric rather than circular orbits. Indeed, all 35 known extrasolar planets with semimajor axes beyond $0.1 \mathrm{AU}$ reside in eccentric orbits (eg. Vogt et al. 2000, Fischer et al. 2000). The circularity of planet orbits, assumed ubiquitous based on our own Solar System, may represent a rare architecture for planetary systems.

Eccentric orbits in planetary systems expose a possible defect in the Copernican principle. Our Solar System may be somewhat unusual in its circular orbits which soften temperature swings to permit the progressive evolution of complex life. The old argument that planetary systems would generally resemble our own may have been circular.

\subsection{HD168443}

HD 168443 exhibits coherent Doppler residuals to the Keplerian fit of the known planet. This G5 star resides $1.5 \mathrm{mag}$ above the zero-age main sequence. It is metal poor with $[\mathrm{m} / \mathrm{H}]=-0.14$ (Carney et al. 1994), and has a mass of $0.95 \mathrm{M}_{\odot}$ (Marcy et al. 1999). The residuals to the Keplerian fit for its inner planet $(\mathrm{P}=$ $58 \mathrm{~d}$ ) exhibited curvature, indicating the presence of another companion (Marcy 
et al. 1999). The new velocities through October 2000 reveal nearly the entire orbit of a second, outer companion (Figure 5). It has a period, $\mathrm{P}=4.8 \pm 0.3 \mathrm{yr}$, $K=290 \pm 20 \mathrm{~m} \mathrm{~s}^{-1}$, eccentricity, $e=0.2$, yielding $M \sin i=16 \mathrm{M}_{\mathrm{JUP}}$.

This system of a Solar-type star and two massive companions is the first of its kind. The outer companion would normally be deemed a 'brown dwarf" by virtue of its minimum mass of $16 \mathrm{M}_{\mathrm{JUP}}$. The inner companion, with minimum mass 6.5 $\mathrm{M}_{\mathrm{JUP}}$, may also have a mass twice its $M \sin i$, rendering it a possible "brown dwarf". Thus, this system apparently contains two unusually massive companions of unclear nature.

This system is remarkable because of the paucity of brown-dwarf companions within $3 \mathrm{AU}$ of Solar-mass stars (Marcy and Benitz 1989, Marcy \& Butler 1998). Indeed, this outer companion is the first companion having $M \sin i$ between $10-80 \mathrm{M}_{\mathrm{JUP}}$ that we have detected in our sample of 1,100 stars.

It is unlikely that the two companions are in fact stars (in face-on orbits), as the system would be dynamically unstable if their masses were above 60 MJUP. (We thank Greg Laughlin for this personal communication.) However the system could remain dynamically stable if $\sin i$ were as low as 0.25 , making the individual masses 4 times their $M \sin i$ values (Laughlin, personal comm). There is no standard theoretical understanding of the origin of two companions having 6-30 MJUP within $3 \mathrm{AU}$ of a star.

Acknowledgments. We would like to acknowledge K.Apps, Greg Laughlin, R.Noyes, T.Brown, D.Charbonneau, M.Mayor, D.Queloz, and S.Udry for many useful discussions. We thank the National Science Foundation, NASA, and Sun Microsystems for support. We thank the W.J.Keck foundation, Lick Observatory, and their telescope assignment committees.

\section{References}

Bundy, K., \& Marcy, G.W. 2000, PASP, 112, in press

Butler, R. P., Marcy, G. W., Williams, E., McCarthy, C., Dosanjh, P., \& Vogt, S. S. 1996, PASP, 108, 500

Butler, R. P., Marcy, G. W., Fischer, D. A., Brown, T. M., Contos, A. R., Korzennik, S. G., Nisenson, P., \& Noyes, R. W. 1999, ApJ, 526, 916

Butler, R. P., Marcy, G. W., Vogt, S.S., \& Fischer, D. A. 2000, this volume.

Charbonneau, D., Brown, T. M., Latham, D. W., \& Mayor, M. 2000, ApJ, 529, L49

Carney, B.W., Latham, D,W., Laird, J.B., \& Aguilar, L.A. 107, 2240

Cumming, A., Marcy, G., \& Butler, R.P. 1999, ApJ, 526, 890

Fischer, D. A., Marcy, G. W., Butler, R. P., Vogt, S. S., \& Apps, K. 1999, PASP, 111, 50

Fischer, D. A., Marcy, G. W., Butler, R. P., Vogt, S. S., \& Apps, K. 2000, ApJ, in press,

Hatzes, A., \& Cochran, W.D. et al. 2000, preprint.

Henry, G. W., Marcy, G. W., Butler, R. P., \& Vogt, S. S. 2000a, ApJ, 529, L45

Laughlin, G., \& Adams, F.C. 1999, ApJ, 526, 881

Levison, H. F., Lissauer, J. J., \& Duncan, M. J. 1998, AJ, 116, 1998

Lloyd, J., \& Patience, J. et al. 2000, this volume.

Marcy, G.W., \& Benitz, K.J. 1989, ApJ, 344, 441

Marcy, G. W., \& Butler, R. P. 1998, ARA\&A, 36, 57 
Marcy, G. W., Butler, R. P., Vogt, S. S., Fischer, D. A., \& Liu, M. 1999 ApJ, 520, 239

Marcy, G. W., Cochran, W. D., \& Mayor, M. 2000, in Protostars and Planets IV, ed V. Mannings, A. P. Boss \& S. S. Russell (Tucson: University of Arizona Press), p.1285

Queloz, D., Eggenberger, A., Mayor, M., Perrier, C., Beuzit, J. L., Naef, D., Sivan, J. P., \& Udry, S. 2000, A\&A, 359, L13

Rivera, E.J., \& Lissauer, J.J. 2000, ApJ, 530, 454

Saar, S. H., Butler, R. P., \& Marcy, G. W. 1998, ApJ, 498, L153

Saar, S. H., Steven, H., \& Fischer, D. 2000, ApJ, 534 L105

Udry, S., Mayor, M., Naef, D., Pepe, F., Queloz, D., Santos, N. C., Burnet, M., Confino, B., \& Melo, C. 2000, A\&A, , 356, 590

Udry, S., Mayor, M., Naef, D., Pepe, F., Queloz, D., \& Santos, N. C. 2000, this volume. Vogt, S. S. 1987, PASP, 99, 1214

Vogt, S. S., Marcy, G. W., \& Butler, R. P., \& Apps, K. 2000, ApJ, 536, 902

Weidenschilling, S.J., \& Marzari, F. 1996, Nature, 384, 619 